\title{
Duet: Static Analysis for Unbounded Parallelism
}

\author{
Azadeh Farzan and Zachary Kincaid \\ University of Toronto
}

\begin{abstract}
DuET is a static analysis tool for concurrent programs in which the number of executing threads is not statically bounded. DUET has a modular architecture, which is based on separating the invariant synthesis problem in two subtasks: (1) data dependence analysis, which is used to construct a data flow model of the program, and (2) interpretation of the data flow model over a (possibly infinite) abstract domain, which generates invariants. This separation of concerns allows researchers working on data dependence analysis and abstract domains to combine their efforts toward solving the challenging problem of static analysis for unbounded concurrency. In this paper, we discuss the architecture of DUET as well as two data dependence analyses that have been implemented in the tool.
\end{abstract}

\section{Introduction}

Verification of concurrent programs is a notoriously challenging problem. The difficulty arises from the fact that the size of the control space of a concurrent program is exponential in the number of executing threads, which makes direct analysis of the control space infeasible. The problem is even more difficult for programs where the number of executing threads is not bounded: in this case, the control space is infinite.

In this paper, we present DUET, a static analysis tool for analyzing programs with unbounded parallelism. DUET is based on the general philosophy that general invariant generation can be reduced to data-dependence analysis. This philosophy is particularly interesting in the case of programs with unbounded parallelism, since in this setting invariant generation is a formidable problem but data-dependence analysis is tractable. In this paper, we expound on the philosophy of DUET. We describe its architecture and describe two different analyses implemented in the tool 415. Finally, we present experimental results demonstrating the efficacy of our tool on a set device drivers.

\section{Overview}

DUET is based on the idea that much of the essential behaviour of a program is captured by the data flow of the program. Consider the program below, in which unboundedly many copies of the thread Thread $i$ execute in parallel. A data flow graph (DFG) for this program is pictured to its right. The edges of this DFG match each read of a variable with the writes that may reach it: for example, the edge from $c:=1$ to $x:=x+c$ labeled $c$ indicates that the value of $c$ may 
flow from $\mathrm{c}:=1$ to $\mathrm{x}:=\mathrm{x}+\mathrm{c}$ (and thus, $\mathrm{c}$ may be 1 at $\mathrm{x}:=\mathrm{x}+\mathrm{c}$ ). Note that some edges in this graph go in the opposite direction of control flow (e.g., the double-headed edge between $\mathrm{x}:=\mathrm{x}+\mathrm{c}$ and $\mathrm{x}:=\mathrm{x}-\mathrm{c}$, which indicates a pair of edges, one in each direction). The value of $x$ may flow from $x:=x-c$ to $\mathrm{x}:=\mathrm{x}+\mathrm{c}$ because these instructions may be executed by different threads. Notice also that several interesting edges do not exist: there is no x-labeled loop on $\mathrm{x}:=\mathrm{x}-\mathrm{c}$; Lock 1 enforces the atomicity of the lock block, which breaks this data flow; an occurrence of $\mathrm{x}:=\mathrm{x}+\mathrm{c}$ must always be executed between any two $\mathrm{x}:=\mathrm{x}-\mathrm{c}$ instructions.

A data flow graph represents a system of constraints that can be solved automatically (using standard techniques) to yield program invariants 5]. This is the essential idea of the DuET tool: since DFGs can be used to compute invariants, invariant generation can be reduced to constructing a DFG for a program. Moreover, since data flow captures an essential feature of program behaviour, this strategy is often sufficient to prove properties of interest. For the example program above, DUET is

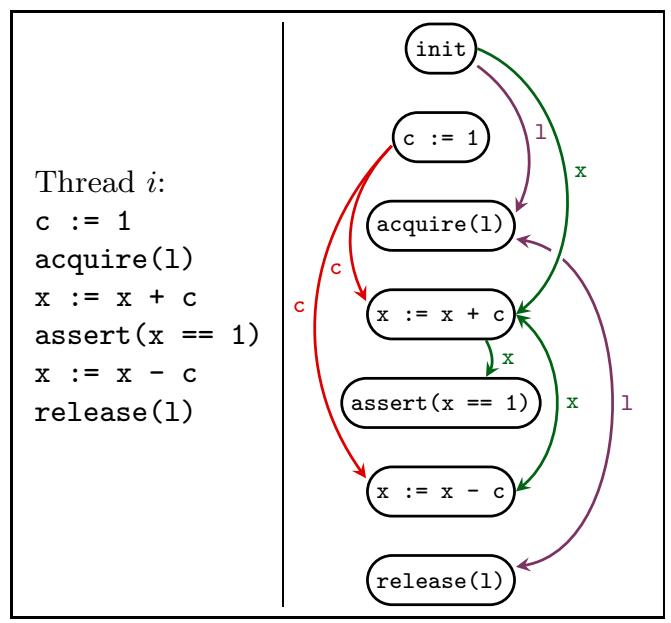
able to prove that assertion always holds by interpreting the DFG over an interval abstract domain.

\section{Duet's Architecture}

The high-level architecture of DUET is pictured in Figure 1, We will begin by describing the basic work-flow of DUET and then discuss each of the component modules in more detail.

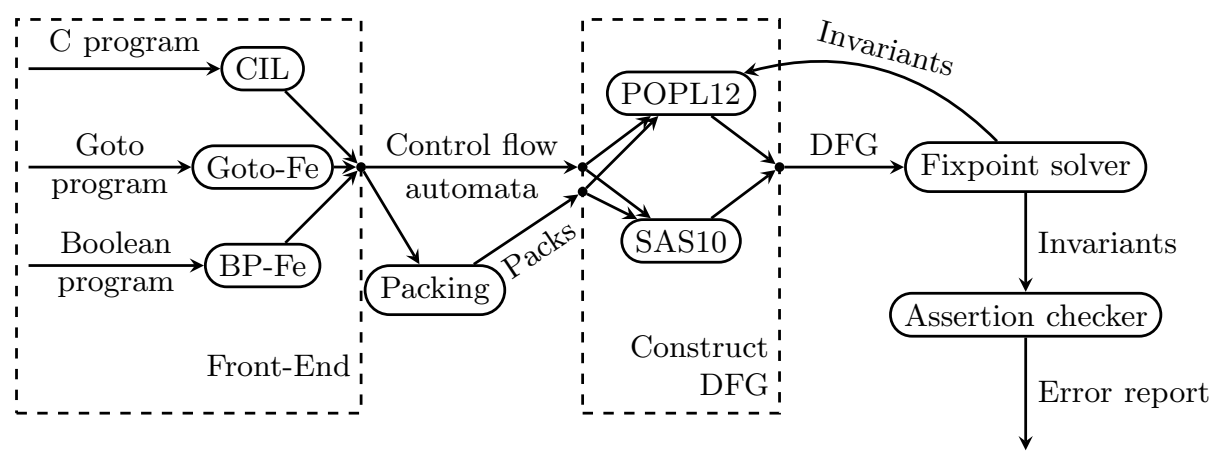

Fig. 1. DuET's architecture 
First, one of the three front-end modules (CIL, Goto-Fe, BP-Fe) takes a (C, Goto, or Boolean) program as input and produces a system of control flow automata, with one automaton for each thread of the program. Next, a variable packing algorithm determines a set of packs (a pack is a set of semantically related variables, according to some heuristic). The set of packs and the system of control flow automata are fed into one of the two construct DFG modules (POPL12 or SAS10), which uses a data dependence analysis to construct a DFG for the program. The fixpoint solver interprets the DFG over some abstract domain to compute a set of invariants, which are passed to the assertion checker (in the case of POPL12, the invariants may alternatively be fed back into the DFG construction module). The assertion checker uses the invariants to determine check which assertions are safe and which may fail, and generates an error report.

\subsection{Front-End}

DUET accepts three types of inputs: (1) C programs using pthreads library for thread operations (using the CIL front-end [9]), (2) Boolean programs, or (3) goto programs, as produced by the GOTO-CC C/C++ front-end (part of the CPROVER project [1]). The front-end transforms these programs into a common form, namely a system of control flow automata. It also annotates the program with assertions, according to user input (for example, an assertion assert $(p !=0)$ is generated for each access path of the form $* \mathrm{p}$, if the -check-null-pointer option is set).

\subsection{Variable Packing}

In the DFGs implemented in DUET, each edge is labeled by a pack rather than a variable. A pack is a set of variables that may be related to one another. We may think of a DFG edge labeled by a pack as "carrying" a value for each variable in that pack (which allows an abstract domain to correlate variables belonging to a pack). The case where edges are labeled by variables is the special case where all packs are singleton sets. The packing module computes a set of packs from an input program, and passes those packs to the DFG construction phase. The choice of the abstract domain (non-relational vs relational) determines the choice of the packing algorithm used (there are currently two options in DUET).

\subsection{DFG Construction}

A DFG construction module takes a system of control flow automata and a set of packs (and in the case of POPL12, a set of invariants), and constructs a DFG representation of the program. There are currently two strategies for constructing DFGs that are implemented in DUET, which we describe below.

Nested locks (SAS10). In [4, we leverage reachability results for concurrent programs communicating via nested locks [6] to develop a compositional technique for solving bitvector analysis problems. Data-dependence analysis can be formulated as a bitvector problem, so we may use this technique to construct DFGs. Our method computes a summary for each thread describing its behaviour and then

${ }^{1}$ http://www .cprover.org/boolean-programs/ 
composes the summaries to compute a DFG for the program. This compositional approach enables our analysis to be sound and precise (it computes meet-overfeasible-paths solutions) even when the number of executing threads in unbounded.

Global variables (POPL12). A more challenging (and also strictly more general) program model uses global variables for synchronization rather than locks. This model is difficult because it requires circular reasoning: in order to perform a reasonably accurate data dependence analysis to construct a DFG we need invariants for the synchronization variables, and in order to compute invariants for the synchronization variables we need to construct a DFG.

In [5], we present an approach based placing the DFG construction module (POPL12) and the fixpoint solver into a feedback loop. The DFG and the invariants are iteratively coarsened as the algorithm progresses (that is, the invariants become weaker, and more edges are added to the DFG) until a fixpoint is reached. When a fixpoint is reached, the invariants overapproximate the dynamic behaviour of the program in question.

\subsection{Fixpoint Solver}

The fixpoint solver module interprets a DFG over an abstract domain to yield program invariants. It accomplishes this by computing a weak topological order on the DFG and repeatedly evaluating DFG nodes over the chosen abstract domain until a fixpoint is reached, as in [3]. DUET employs the abstract domains implemented in APron [2] for this task.

\subsection{Assertion Checker}

Finally, the assertion checker module iterates over the DFG vertices: for each assertion vertex, we determine whether the invariant at that location (computed by the fixpoint solver) implies the assertion using the APRON [2] library. If the check fails, the assertion may fail, and we add it to the error report.

\section{Experiments}

We used a benchmark suite of 15 Linux device drivers to evaluate DuET. Since a driver may have arbitrarily many clients, these programs exhibit unbounded parallelism. Table 1 presents the result of running DUET on a collection of 15 Linux device drivers. These drivers are all written in C, and include infinite data (such as integer types).

With an interval analysis, DUET manages to prove most of the assertions correct (1312 out of a total 1597), and does so in 13 minutes. DUET's performance using an octagon analysis is slightly worse, proving 1277 assertions correct in 90 minutes.

Most false positives for DUET appear to be caused by one of two reasons: imprecision in the abstract domain (e.g. lack of a precise enough abstraction to handle zero-terminated arrays that are used in most of these drivers), and imprecision in how DUET handles the treatment of spinlocks in goto programs (due to the imprecision in the alias analysis that for lock variables). Neither of 
Table 1. DuET's Performance on Integer Programs with unbounded parallelism, run on an $3.16 \mathrm{GHz}$ Intel(R) Core 2(TM) machine with 4GB of RAM

\begin{tabular}{|l|c||c|c||c|c|}
\hline \multirow{2}{*}{ Device Drivers } & \multirow{2}{*}{ \#assertions } & \multicolumn{2}{|c||}{ DUET: Interval Analysis } & \multicolumn{2}{c|}{ DUET: Octagon Analysis } \\
\cline { 3 - 6 } & & safe & time & safe & time \\
\hline \hline i8xx_tco & 90 & 75 & $1 \mathrm{~m} 51 \mathrm{~s}$ & 71 & $1 \mathrm{~m} 25 \mathrm{~s}$ \\
\hline ib700wdt & 75 & 64 & $30 \mathrm{~s}$ & 64 & $20 \mathrm{~s}$ \\
\hline machzwd & 87 & 73 & $39 \mathrm{~s}$ & 67 & $14 \mathrm{~m} 44 \mathrm{~s}$ \\
\hline mixcomwd & 91 & 72 & $22 \mathrm{~s}$ & 74 & 25 \\
\hline pcwd & 240 & 147 & $2 \mathrm{~m} 43 \mathrm{~s}$ & 145 & $23 \mathrm{~m} 48 \mathrm{~s}$ \\
\hline pcwd_pci & 204 & 187 & $2 \mathrm{~m} 18 \mathrm{~s}$ & 188 & $2 \mathrm{~m} 59 \mathrm{~s}$ \\
\hline sbc60xxwdt & 91 & 77 & $28 \mathrm{~s}$ & 69 & $11 \mathrm{~m} 27 \mathrm{~s}$ \\
\hline sc520_wdt & 85 & 71 & $28 \mathrm{~s}$ & 65 & $13 \mathrm{~m} 20 \mathrm{~s}$ \\
\hline sc1200wdt & 77 & 66 & $34 \mathrm{~s}$ & 66 & $33 \mathrm{~s}$ \\
\hline smsc37b787_wdt & 93 & 80 & $47 \mathrm{~s}$ & 80 & $47 \mathrm{~s}$ \\
\hline w83877f_wdt & 92 & 78 & $29 \mathrm{~s}$ & 72 & $13 \mathrm{~m} 24 \mathrm{~s}$ \\
\hline w83977f_wdt & 101 & 90 & $34 \mathrm{~s}$ & 82 & $34 \mathrm{~s}$ \\
\hline wdt & 99 & 88 & $25 \mathrm{~s}$ & 86 & $25 \mathrm{~s}$ \\
\hline wdt977 & 88 & 77 & $27 \mathrm{~s}$ & 75 & $28 \mathrm{~s}$ \\
\hline wdt_pci & 84 & 67 & $33 \mathrm{~s}$ & 66 & $5 \mathrm{~m} 33 \mathrm{~s}$ \\
\hline \hline total & 1597 & 1312 & $13 \mathrm{~m} 9 \mathrm{~s}$ & 1270 & $90 \mathrm{~m} 21 \mathrm{~s}$ \\
\hline
\end{tabular}

these sources of imprecision is due to a fundamental limitation of the analysis technique proposed in this paper (or related to concurrency). But, they hint on the idea that more precise alias analysis techniques (for concurrent code) and better abstract domains could hugely benefit the false positive rate of DUET.

Interval vs Octagon Analysis. The table on the right compares the results of interval and octagon analyses in DuET over the driver benchmarks (same experiments as in Table 1. Octagon analysis performs worse than inter-

\begin{tabular}{|c|c|c|c|}
\hline & \multicolumn{2}{|c|}{$\mathrm{OCT}$} \\
\hline & & safe & unsafe \\
\hline$\theta$ & safe & 1267 & 45 \\
\hline & unsaf & 3 & 282 \\
\hline
\end{tabular}
val analysis, however, it manages to prove 3 assertions safe that interval analysis fails. Octagon analysis is very sensitive to the variable packing algorithm. Investigating more sophisticated packing algorithms is a topic of our future work.

\subsection{Boolean Programs}

Although Boolean programs are not the intended target of DUET, we performed a set of experiments over existing Boolean program benchmarks for two reasons: (1) to compare with two recent approaches [87] for verification of concurrent Boolean programs with unboundedly many threads, and (2) there is no aliasing present in Boolean programs, which limits the scope of implementation-related imprecision for a better evaluation of the core method.

Even though DuET can directly analyze the original device driver codes (i.e. does not require a predicate abstraction phase), we chose to compare with the existing tools [8] on the Boolean abstractions (for which these tools were designed), to present a more fair comparison. We compare DUET against two recent algorithms that handle Boolean programs with unbounded parallelism: dynamic cutoff detection (DCD) from [7], as implemented in Boom, and linear interfaces (LI) from [8], 
as implemented in Getafix. We compared these tools against the benchmarks provided by the authors. It is important to note that both tools are capable of finding counterexamples to definitively declare an assertion unsafe, whereas, when DUET fails to produce strong enough invariants to prove an assertion correct, it is not clear whether the assertion is incorrect or DUET failed to prove it correct. The timeout is 5 minutes in all cases.

\begin{tabular}{|c|c|c|c|c|c|}
\cline { 3 - 6 } \multicolumn{2}{c|}{} & \multicolumn{4}{c|}{ LI } \\
\cline { 3 - 6 } \multicolumn{2}{c|}{} & safe & unsafe & timeout & unknown \\
\hline & safe & 1289 & 0 & 267 & 957 \\
\hline \multirow{2}{|c|}{ unknown } & 54 & 247 & 23 & 579 \\
\hline & timeout & 0 & 0 & 0 & 0 \\
\hline
\end{tabular}

The table on the left presents the results of comparing DUET against LI over LI benchmarks. Columns/ rows titled safe, unsafe, and timeout are self-explanatory. An "unknown" response from DuET means that the invariants were not strong enough to prove the assertion safe, and an "unknown" response from LI means that neither a counter example was found nor the program was proved safe. DUET substantially outperforms LI; for example, there were 957 assertions proved safe by DUET that were declared unknown by LI, where as LI could only prove safe 54 of DUET's unknown cases.

The table on the right presents the results of comparing against DCD over DCD benchmarks. Again, DuET substantially outperforms DCD in proving assertions correct; 39 more assertions (out of 58) are proved correct by DUET.

\begin{tabular}{|c|c|c|c|c|}
\cline { 3 - 5 } \multicolumn{2}{c|}{} & \multicolumn{3}{c|}{ DCD } \\
\cline { 2 - 5 } \multicolumn{2}{c|}{} & safe & unsafe & timeout \\
\hline \multirow{2}{|c|}{ safe } & 19 & 0 & 39 \\
\hline & unknown & 0 & 203 & 2 \\
\hline & timeout & 0 & 7 & 0 \\
\hline
\end{tabular}

\section{References}

1. Alglave, J., Kroening, D., He, N., Ranjan, A., Seghir, N., Tautschnig, M.: CPROVER Project (November 2011)

2. Jeannet, B., Miné, A.: Apron: A library of numerical abstract domains for static analysis. In: Bouajjani, A., Maler, O. (eds.) CAV 2009. LNCS, vol. 5643, pp. 661667. Springer, Heidelberg (2009)

3. Bourdoncle, F.: Efficient chaotic iteration strategies with widenings. In: Pottosin, I.V., Bjorner, D., Broy, M. (eds.) FMP\&TA 1993. LNCS, vol. 735, pp. 128-141. Springer, Heidelberg (1993)

4. Farzan, A., Kincaid, Z.: Compositional bitvector analysis for concurrent programs with nested locks. In: Cousot, R., Martel, M. (eds.) SAS 2010. LNCS, vol. 6337, pp. 253-270. Springer, Heidelberg (2010)

5. Farzan, A., Kincaid, Z.: Verification of parameterized concurrent programs by modular reasoning about data and control. In: POPL, pp. 297-308 (2012)

6. Kahlon, V., Ivančić, F., Gupta, A.: Reasoning about threads communicating via locks. In: Etessami, K., Rajamani, S.K. (eds.) CAV 2005. LNCS, vol. 3576, pp. 505-518. Springer, Heidelberg (2005)

7. Kaiser, A., Kroening, D., Wahl, T.: Dynamic cutoff detection in parameterized concurrent programs. In: Touili, T., Cook, B., Jackson, P. (eds.) CAV 2010. LNCS, vol. 6174, pp. 645-659. Springer, Heidelberg (2010)

8. La Torre, S., Madhusudan, P., Parlato, G.: Model-checking parameterized concurrent programs using linear interfaces. In: Touili, T., Cook, B., Jackson, P. (eds.) CAV 2010. LNCS, vol. 6174, pp. 629-644. Springer, Heidelberg (2010)

9. Necula, G.C., McPeak, S., Rahul, S.P., Weimer, W.: Cil: Intermediate language and tools for analysis and transformation of c programs. In: CC, pp. 213-228 (2002) 